\title{
Numerical Investigation of Multi-scale Characteristics of Single and Multi-layered Woven Structures
}

\author{
Hatim Alotaibi ${ }^{1} \cdot$ Masoud Jabbari $^{1} \cdot$ Chamil Abeykoon ${ }^{2} \cdot$ Constantinos Soutis ${ }^{2,3}$
}

Received: 30 July 2021 / Accepted: 6 January 2022 / Published online: 24 January 2022

(c) The Author(s) 2022

\begin{abstract}
Resin flow through multi-ply woven fabrics is affected by the fibre orientation and laminate stacking sequence during the impregnation process. This is characterised by permeability, which measures the ability of transferring fluids within a 2D or 3D layered woven fibre architecture (i.e., through a porous medium). The work aims to investigate the feasibility of characterising macro-scale flow permeability via the micro-meso-scale (dual-scale) permeability across and along woven yarns, with different structures of yarn nesting, nonshifting, and ply orientation. The permeability characterisation is performed using AnsysFluent software package where textile architectures and resin flow in porous media are simulated. The results show that in- and out-plane permeability of the nested, non-shifted and oriented single-ply woven preforms are different than that corresponding to multi-layered plates, making them only applicable for dual-scale permeabilities. However, with a number of plies in the multi-ply woven fabrics - e.g., 9-ply and 5-ply, for in- and out-of-plane flows, respectively - the dual-scale permeabilities can be extended to macro-flow making them applicable at all scales (multi-scale flow). The calculated in-plane multi-scale permeabilities are then used in the 2D simulations and compared with the analytical solution of the Darcy's equation, which resulted in a very good agreement.
\end{abstract}

Keywords Permeability $\cdot$ Dual-scale porous media $\cdot$ Multi-scale flow $\cdot$ Liquid composite moulding

\section{Introduction}

Resin impregnation during liquid composite moulding (LCM) processes has been an area of interest over several decades. Mainly vacuum assisted resin transfer moulding (VARTM) and resin transfer moulding (RTM) are typical composite manufacturing processes for

Masoud Jabbari

m.jabbari@manchester.ac.uk

1 Department of Mechanical, Aerospace and Civil Engineering, The University of Manchester, Manchester M13 9PL, UK

2 Northwest Composites Centre, Department of Materials, The University of Manchester, Manchester M13 9PL, UK

3 Aerospace Research Institute, The University of Manchester, Manchester M13 9PL, UK 
aerospace, aeronautical, and automotive applications due to their applicability for high fibre volume plies, repeatability, cost-effectiveness, good surface finish, and complex shapes [1-4]. LCM processes for woven-fabric preforms involve the flow of liquid resin permeating through fibrous media. Although the single-phase Darcy law is valid in many cases, it is however proved (experimentally and numerically) that presence of different length scales (micro-, meso-, and macro-level) will change the resin impregnation. This makes a composite preform a multi-scale porous medium in which the resin will usually fill the macroscopic pores between fibre tows much faster than it saturates the microscopic pores inside the fibre tows [5, 6] - see Fig. 1. Generally, depending on the scale of analysis, properties at the smaller scales of the material are usually homogenised, and any changes at these scales are ignored [7]. This flow behaviour is a crucial parameter to predict fibre preforms saturation and void formation. This has motivated researchers to investigate permeability and porosity phenomena in single- or dual-scale fibrous media. A considerable amount of contributions have been made to this field in terms of experiments [8-11], analytical models [12-16], and numerical simulations [17-21]. In terms of numerical approaches, there has been numerous work using finite element, finite volume, finite difference, and Lattice Boltzmann methods that are sufficient in modelling creeping/viscous flow problem in fibrous porous media [17-21].

Permeability measurements depend on flow direction in LCM processes, which can be in longitudinal (in-plane) or through-thickness (out-plane) directions. The throughthickness permeability is a challenging flow parameter to measure and becomes important particularly in VARTM process, since a significant amount of resin would impregnate the preforms through the thickness [22]. Flow across fibre bundles has also been a focus of interest by several previous studies [22-26].

Micro

Fibres/Yarns $[\mu m-m m]$

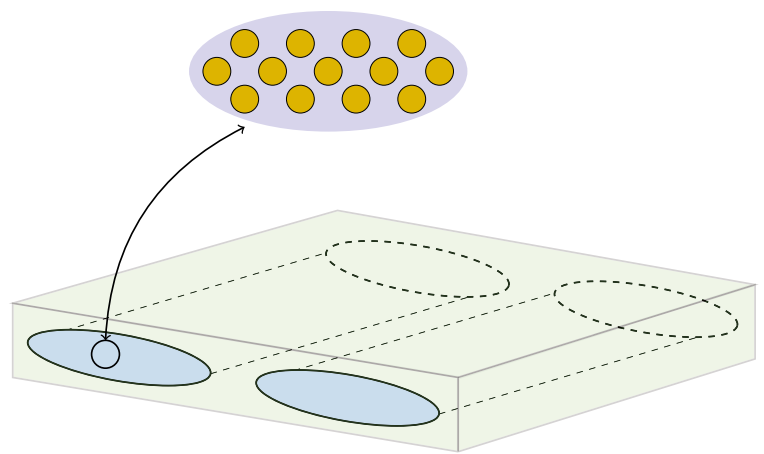

Meso

Yarns/Fabrics $[m m-c m]$

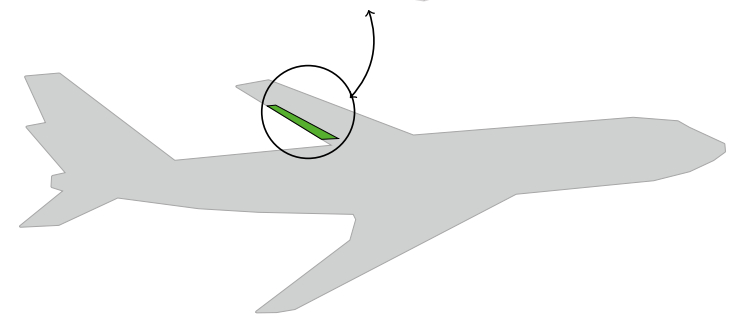

Fig. 1 Multi-scale nature of resin flow in fibrous porous media 
There has been less research published in the open literature that focused on studying the flow behaviour in multi-ply preforms [25, 27-36]. Fang et al. [25] developed a mathematical model to predict out-plane permeability for nested unidirectional (UD) plies. They investigated the effect of number of layers on permeability with consideration of maximum and minimum nesting, and concluded that permeability is relatively high in areas of minimum nesting. Mogavero and Advani [27] conducted an experimental work that studied the influence of variable ply thicknesses on permeability of composite structures. They found that flow progressed entirely within high permeable plies; however, partial progression was observed in thick/rich plies which led to formation of dry spots. Work by Sas et al. [32] focused on the impact of ply orientations or misaligned stacked UD fabrics on through-thickness permeability. The authors performed experimental and numerical analyses. Their results showed that through-thickness permeability is increased in high angles (45- and 90-degrees) of the oriented plies, but with insignificant change for the slightly shifted plies or the low-angled (5-degree) oriented plies. Swery et al. [31] carried out a number of flow simulations using Ansys-CFX to predict in-plane permeabilities for multi-layer woven fabrics at various fibre volume fractions of $25 \%, 34 \%$ and $47 \%$. The study considered non-shifted and nested stacking sequences, which played a part in impacting in-plane permeabilities, as it showed a higher flow resistance, e.g., $33 \%$ for the nested plies versus the non-shifted plies at a low fibre volume fraction $25 \%$.

It should be noted that the numerical studies in literature such as [31, 32] did not take into account the intra-tow flow for the multi-ply preforms, and this means adopting the assumption of solid tows, hence a single-scale flow. Stacking sequences with different ply orientations and their effects on dual-scale permeability in transverse (perpendicular) and longitudinal (axial) directions, have not been extensively explored in terms of numerical simulations. Moreover, the uncertainty of upscaling permeability from micro- to macro-scale, and appropriate choice of representative elementary volume (RVE) are still not accurately investigated in the current open literature. Therefore, this paper is aiming to characterise dual-scale permeability across and along woven tows for single- and multi-ply cells. This will include the effect of ply nesting, non-shifting (zero-offset), and ply orientation, and set a benchmark for a single-/multiply unit cell (meso-scale) applicability range as a RVE of the laminated woven fabrics (macro-scale) - see Fig. 2.

Fig. 2 Permeability (flow analysis) upscaling in LCM, and the scope of current research (dualscale/multi-plies)

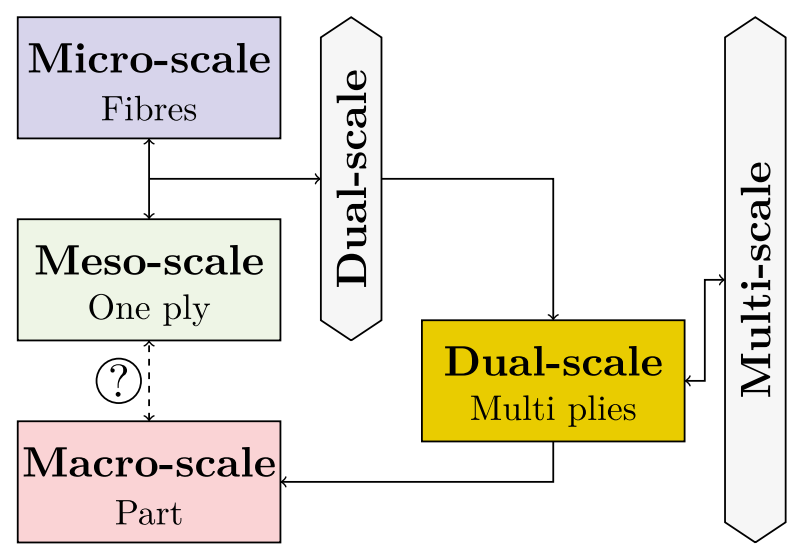




\section{Numerical Approach}

An integrated design tool provided by the commercial software package Ansys is selected to model textiles with different geometries, perform meshing, and also to simulate the dualscale flow. Fig. 3 presents the tool steps of the numerical approach to dual-scale permeability modelling that has been set up in Ansys-Fluent.

\subsection{Porous Model}

Flow simulations are performed in a unit cell of single and multi-ply fabric. This is computed by Fluent solver, wherein the Navier-Stokes $(\mathrm{N}-\mathrm{S})$ equations are employed. AnsysFluent works for regular and complex fabric architectures and offers diverse selection of models. A porous model is one of these offered tools, during which an additional source term is added to the N-S equations. This allows modelling inter-tow flow together with intra-tow flow [19-21]. The conservation of momentum and mass (continuity) equations for laminar viscous flows in an inertial frame of reference (a stationary frame) is presented below:

$$
\frac{\partial}{\partial t}(\rho u)+\nabla \cdot(\rho u u)=-\nabla P+\mu \nabla^{2} u+\rho g+S
$$

Fig. 3 Computation procedures from textiles modelling to flow simulations

\section{Pre-processing}

- Create geometry

- Generate mesh

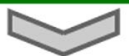

\section{Solver (Fluent)}

Set-up (steady state)

- Model: laminar, viscous flow

- Materials: flow density and viscosity

- Set cell zone conditions:

- identify porous zones (yarns)

o input intra-two permeabilities and porosity

- Set boundary conditions

Solution

- Specify solution and discretisation methods

- Set convergence monitor

- Solution initialisation

\section{Post-processing}

- Analysis of results 


$$
\frac{\partial \rho}{\partial t}+\nabla \cdot(\rho u)=0
$$

where $u$ is the average velocity vector, $\nabla P$ is the pressure gradient vector, $\rho g$ is the body force term, $\mu$ is the viscosity of the fluid, $\tau$ is the stress tensor, $S$ is the source term, and $\nabla \cdot(\rho u u)$ is the convective acceleration term. With the assumptions of steady, incompressible and creeping flow of a Newtonian fluid, the momentum and continuity equations are reduced to:

$$
\begin{gathered}
0=-\nabla P+\mu \nabla^{2} u+S \\
\nabla \cdot u=0
\end{gathered}
$$

Since the flow will behave in a laminar manner (based on the assumptions made), this source term would consist of a viscous loss term as shown below

$$
S=-\frac{\mu}{K_{t}} u
$$

Here, the intra-tow flow or the micro-permeability tensor $\left(K_{t}\right)$ is calculated using the available analytical models [12-16] based on intra-tow porosity and fibre radius. Hence, inserting Eq. (5) into Eq. (3), leads to the so-called Stokes-Brinkman equation that accounts for the fluid flow within free-flow region (inter-tow) and porous region (intra-tow). This will enable the computation of the volume-averaged velocity vector $(u)$ with a given inlet boundary condition pressure. Subsequently, the computed $u$ that considered the dual-scale flow, is required to solve the global permeability (micro- and meso-regions). Thus, the generalised form of the well-known Darcy's law, is used to calculate the global permeability tensor $\left(K_{o}\right)$ of the dual-scale fibre preforms.

$$
u=-\frac{K_{o}}{\mu} \nabla P
$$

\subsection{Textile Models}

Woven models are created by Ansys DesignModeler as a part of an integrated environment. This modelling tool provides multiple functions that are efficient in customising designs for fluid flow problems. These models include nested, non-shifted, and oriented-ply unit cells. The nesting phenomena would show a maximum ply shift, while the non-shifting (zerooffset) denotes the direct ply stacking on top of each other with an identical orientation (cross-ply) of the plain weave, in particular, $0^{\circ}$ (warp) and $90^{\circ}$ (weft). This is different for oriented-ply situations, in which this work follows quasi-isotropic woven fabrics lay-ups in a sequential form of $0^{\circ}$ (warp), $90^{\circ}$ (weft) and $\pm 45^{\circ}$. Flow simulations are executed on several fabric plies for each stacking method. The number of plies begins with a single-ply (1 ply) and increments in an arithmetic sequence of odd numbers until reaching 15 stacked plies. These plies are designed with various aggregate (overall) porosities $\phi_{o}\left(=1-V_{f}\right)$, $40 \%, 50 \%$ and $60 \%$. This is done by changing the yarn cross-sectional shape, and mainly the major axis size, which falls into the practical range for composite materials/manufacturing [19]. A ply lay-up gap is counted as $0.025 \mathrm{~mm}$ to avoid overlapping mesh issues, and 


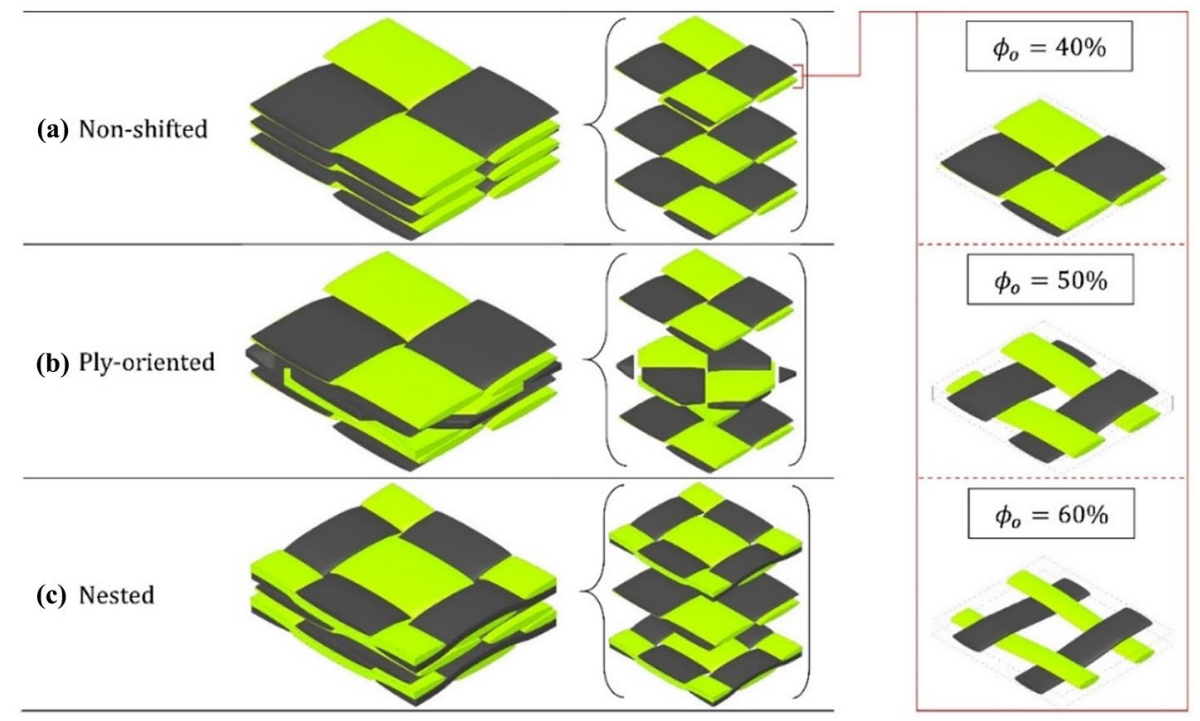

Fig. 4 Woven models: (a) non-shifted ply, (b) oriented ply $\left(0^{\circ}\right.$ (warp), $90^{\circ}$ (weft) and $\pm 45^{\circ}$ ), and (c) nested ply

to attain proper numerical calculations across the grid cells (control volumes). Figure 4 illustrates the woven models with different stacking methods, and also shows a schematic example of unit cells with different fibre volume fractions. The dimensional details of the woven fabric geometries for various unit cells' aggregate porosities are provided in Table 1.

\subsection{Flow Simulation Boundary Conditions}

Resin flow is driven by a pressure gradient force to pass the flow in-between and through the fibre bundles. In a flow simulation, this will be imposed on a unit cell as an inlet pressure (provided that the pressure at the outlet is set to zero). The top and bottom surfaces as well as the left and right walls are set as no-slip boundary condition. This allows simple implementation/computational efficiency in terms of meshing and shows only an ineffective difference if they were set as periodic boundary condition. Figure 5 illustrates the boundary conditions in a rectangular unit cell (single ply) domain $6.56 \mathrm{~mm} \times 5.66 \mathrm{~mm} \times 0.575 \mathrm{~mm}$ with an extended inlet region $0.5 \mathrm{~mm}$. The other required inputs to the flow model such as density and viscosity are $\rho=1300 \mathrm{~kg} / \mathrm{m}^{3}, \mu=0.15 \mathrm{~Pa} \cdot \mathrm{s}$, respectively, with an injection pressure of $10 \mathrm{kPa}$ at the inlet.

Table 1 Details of the woven fabric geometries for various unit cells' aggregate porosities:

\begin{tabular}{llll}
\hline Parameter & \multicolumn{3}{l}{ Unit cell $\phi_{o}[\%]$} \\
\cline { 2 - 4 } & 40 & 50 & 60 \\
\hline Width warp yarns $[\mathrm{mm}]$ & 2.46 & 1.63 & 0.72 \\
Gap warp yarns $[\mathrm{mm}]$ & 0.33 & 1.16 & 2.07 \\
Width fill yarns $[\mathrm{mm}]$ & 3.10 & 2.05 & 0.90 \\
Gap fill yarns $[\mathrm{mm}]$ & 0.04 & 1.09 & 2.24 \\
\hline
\end{tabular}




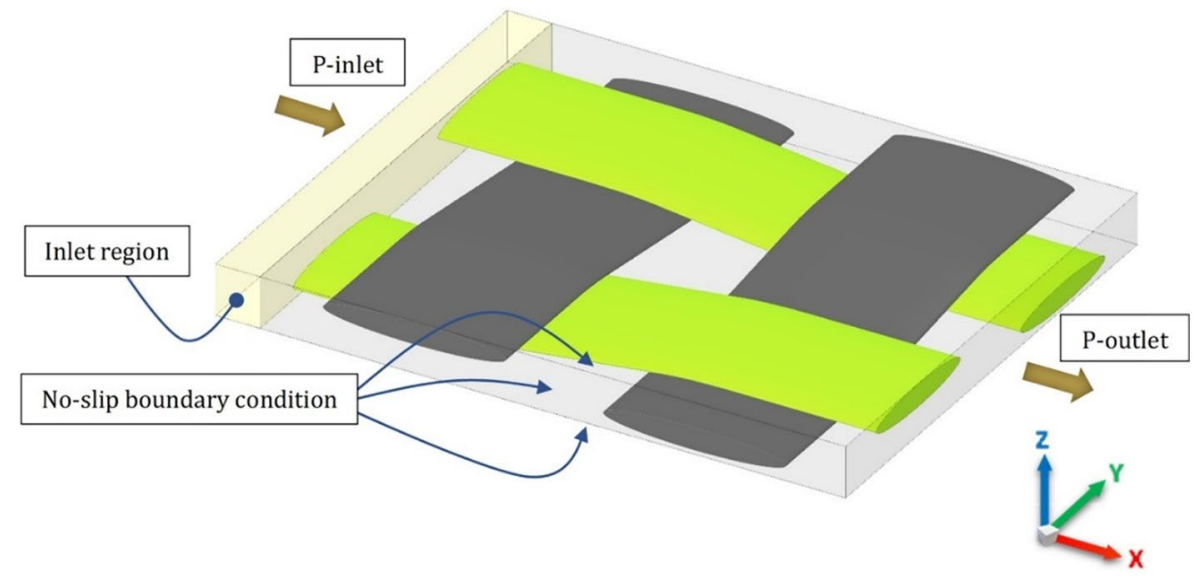

Fig. 5 Unit cell of a single-ply fabric and its boundary conditions (b.c.)

\section{Results and Discussion}

A comparative analysis is conducted for single and different multi-stacked woven fabric/s at various aggregate (total) porosities of $40 \%\left(V_{f}=60 \%\right), 50 \%\left(V_{f}=50 \%\right)$ and $60 \%\left(V_{f}=40 \%\right)$. The aggregate porosity, $\phi_{o}$, incorporates both inter-tow porosity, $\phi_{s}$, and intra-tow porosity, $\phi_{t}$, as follows

$$
\phi_{o}=\phi_{s}+\phi_{t}-\phi_{s} \phi_{t}
$$

The analysis assumes constant intra-tow porosity $\left(\phi_{t}=20 \%\right)$ and hexagonal packed filaments with a radius $R_{f}=10.5 \mu \mathrm{m}$ within the fibre bundle. This also follows the consideration of a homogenous isotropic porous medium, during which the in-plane permeabilities $K_{x x}$ (warp direction) and $K_{y y}$ (weft direction) are roughly equivalent $\left(K_{x x}=K_{y y}=K\right)$. This will be different for through-thickness (out-of-plane) permeability $\left(K_{z z}\right)$, since the fluid flow would penetrate across the fibre bundles for such a plain weave model. All simulations are subject to a mesh analysis, in which results are selected wherever mesh independency is met and that appears after applying refinements to the model. Figure 6 depicts a mesh refinement map for all three models with flow simulations at $\phi_{o}=40 \%$. This begins with a convergence criteria for the residual values (e.g., continuity, velocity, etc.), and this is then followed by more mesh refinements to compare the first simulation of the initial mesh with the one that is based on furthered refined grids, and so forth. An example is illustrated by Fig. 7 for a nested 9-ply woven model where insignificant changes for values of a monitor point (i.e., velocity) are seen above 1,356,186 mesh elements $(N)$, and hence results tend to become independent of mesh. Therefore, this solution technique is used for all the simulations presented in the following.

\subsection{Through-Thickness Dual-Scale Permeability}

Figure 8 demonstrates through-thickness dual-scale permeabilities $\left(K_{z z}\right)$ for different ply stacking methods and various aggregate porosities. All sub-figures are plotted at the same 
Fig. 6 Mesh refinement map of numerical simulations for different fabric structures at $\phi_{o}=40 \%$

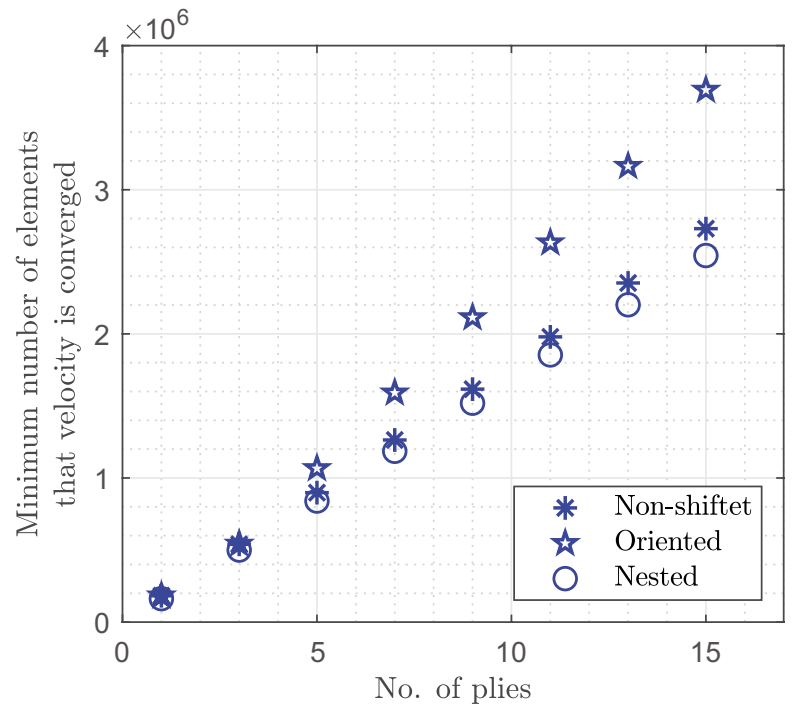

scale for ease of comparison. The results show that the increase in number of plies, has a slight effect on $K_{z z}$ for multi-ply cases. However, it certainly shows that the permeability value for one ply is different than multi-ply structures. Transverse flows through nested and non-shifted plies appear to behave in a similar manner, and this is mostly due to the same warp (x) and weft (y) directions for both cases. This will allow identical flow pass across the fibres (filaments), resulting in a relatively similar influence. In contracts, the oriented-ply case shows higher through-thickness permeabilities for multi-ply stackings. This increase would be ranged from 20 to $35 \%$ at aggregate porosity $\left(\phi_{o}\right)$ values $50 \%$ and $60 \%$, and becomes $17 \%$ for multi-ply woven models with $\phi_{o}=40 \%$. This shows that the oriented-ply woven fabrics provide more flow passages, but this phenomenon reduces in high $V_{f}\left(\phi_{o}=40 \%\right)$.

For each multi-ply stacking method at various $\phi_{o}$, a change in through-thickness dualscale permeability initially evolves and then tends to stabilise at about a certain value. This

Fig. 7 Mesh dependency study for a nested 9-ply woven model at $\phi_{o}=40 \%$

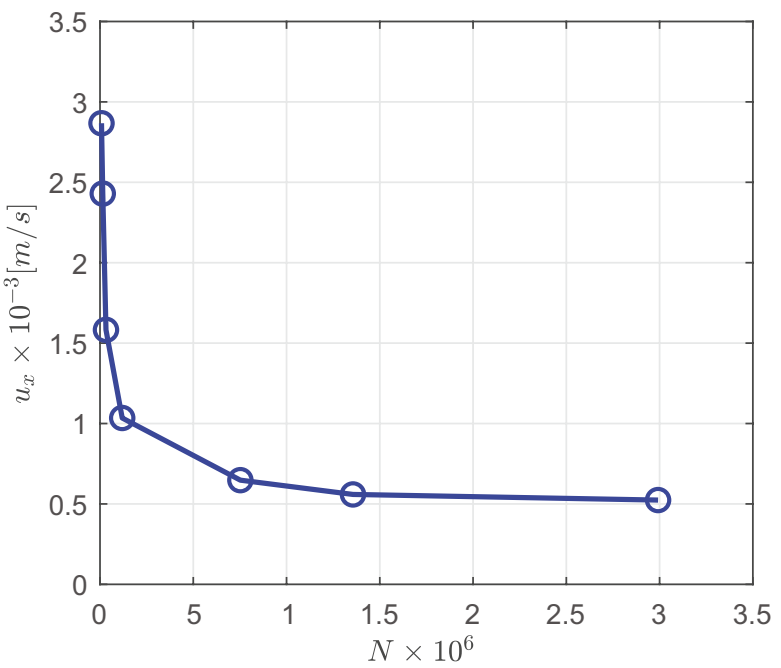


(a) Nested

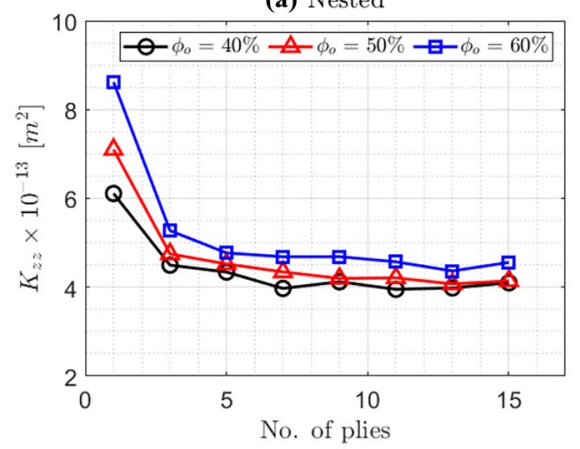

(b) Non-shifted

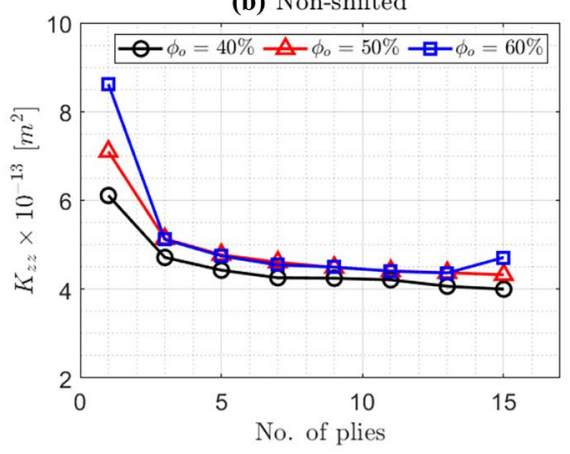

(c) Oriented

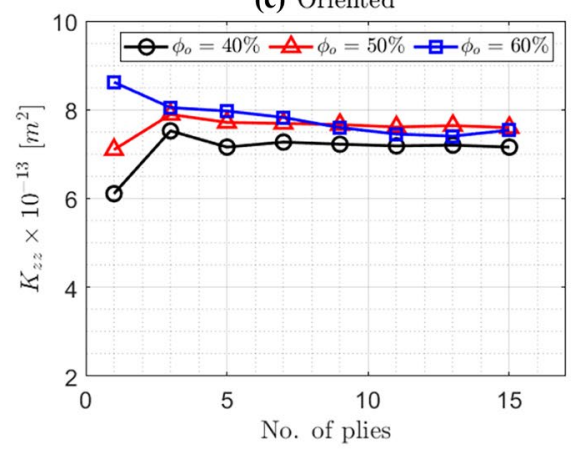

Fig. 8 Through-thickness dual-scale permeabilities for different ply stacking methods and various aggregate porosities: (a) nested, (b) non-shifted, and (c) oriented

means that the initial stabilising point (i.e., meso-permeability) can be taken as a RVE that will yield the macro-permeability value of the entire stacked woven fibre preforms. The results indicate that beyond 5-ply woven fabrics, fairly insignificant change $(1 \%-10 \%)$ in dual-scale permeability, is observed for all through-thickness cases. As for instance, the nested multi-ply woven model at $\phi_{o}=40 \%$ depicts approximately $5.9 \%$ variation, with being 4.34 and $4.10\left(\times 10^{-13} \mathrm{~m}^{2}\right)$ for 5- and 15-ply, respectively. Concerning the single-ply unit cell, the dual-scale permeability of the 5-ply has $33.8 \%$ increase, as this shows 6.11 and $4.34\left(\times 10^{-13} \mathrm{~m}^{2}\right)$ values for 1-ply and 5-ply, respectively. This suggests modelling of filling process parameters within a single-ply woven fabric (still with dual-scale model) cannot be used to predict the macroscopic flow. Nonetheless, this becomes different for multi-ply case, since the results show nonignorable discrepancy in permeability between single-ply and multi-ply unit cells (UCs). Thus, the study stresses that at least a 5-ply UC will be sufficient to represent flow parameters (e.g., permeability) of multiple plies ( $>5$ -ply) of fibre preforms.

\subsection{In-Plane Dual-Scale Permeability}

The in-plane flow study presents different trends compared to the through-thickness analysis. This can be explained by the fact that the in-plane flow behaves across and 
along the fibre bundles, encountering lower resistance to the flow. As seen from Fig. 9, increasing the number of plies shows a gradual increase in dual-scale permeabilities for all cases, and this would tend to level off (and stabilise) beyond 7-ply structure for aggregate porosity of $40 \%$, and beyond 9-ply topology for both porosities of $50 \%$ and $60 \%$. Since the nested and non-shifted multi-ply woven models have the same warp (x) and weft $(\mathrm{y})$ directions, their effect on the in-plane dual-scale permeability $(K)$ are comparable. While this effect is still considerable in terms of dual-scale permeability of a single-ply compared to multi-ply, it becomes even more significant for the oriented woven model. The results show that $K$ values for the nested and non-shifted cases are in the same order of magnitude, and this includes the single- and multi-ply UCs at each aggregate porosity value. On the other hand, this becomes one order of magnitude larger for the oriented ply case, in which the in-plane flow within a single-ply is significantly lower than multi-ply fabrics. This stems from the fact that the angled plies at $\pm 45^{\circ}$ allows more flow passages and a lower viscous resistance. At these angled $\left( \pm 45^{\circ}\right)$ plies, both weft and warp yarns facilitate longitudinal intra-tow flows, while the $0^{\circ}$ warp and $90^{\circ}$ weft yarns (which are the case for nested and non-shifted as well as the single-ply model) offers only longitudinal intra-tow flows within the warp bundles. By virtue of different ply orientations, the in-plane dual-scale flow through a single-ply unit cell would not be appropriate to represent the macro flow characteristics of the multiple plies using dual-scale permeability.

(a) Nested

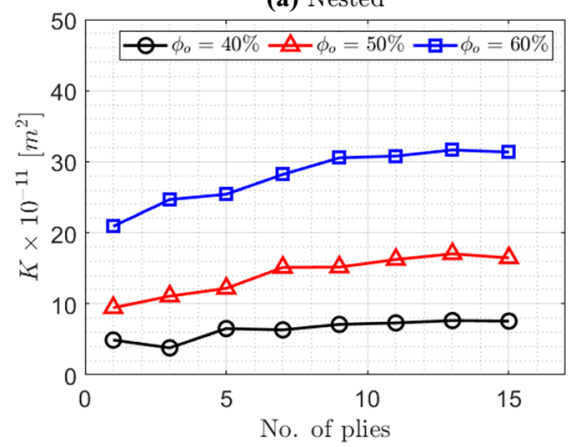

(b) Non-shifted

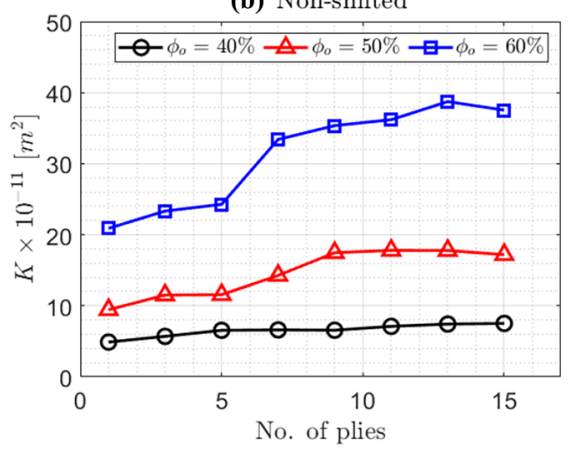

(c) Oriented

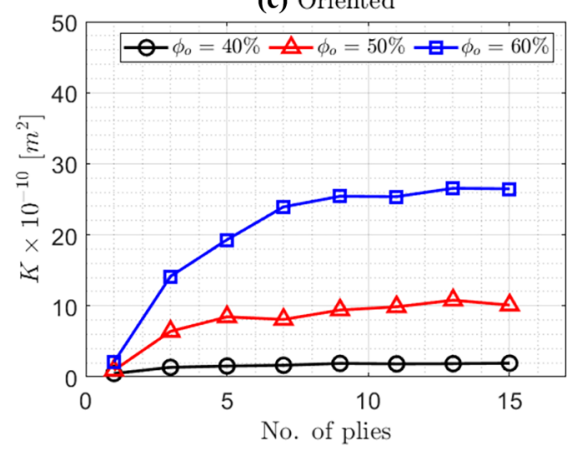

Fig. 9 In-plane dual-scale permeabilities for (a) nested, (b) non-shifted, and (c) oriented ply stacking methods and at various aggregate porosities 
As mentioned earlier, the dual-scale permeability begins with a slight increase and followed by a stable flow behaviour for nested and non-shifted multi-ply unit cells of woven preforms. During these stabilising points, a varying difference can be observed ranging from $3 \%$ to $13.48 \%$ for the UCs at various overall porosity values. The highest variation is captured at high fibre volume fraction of woven fabrics $60 \%\left(\phi_{o}=40 \%\right)$ which describes that the micro-flow (intra-tow flow) plays an important role on the global permeability. Taking an example of the nested multi-ply case at $\left(\phi_{o}=60 \%\right)$, the $K$ shows $\sim 3 \%$ change, e.g., 30.5 and $31.35\left(\times 10^{-11} \mathrm{~m}^{2}\right)$ at 9 - and 15 -ply, respectively. Accordingly, this highlights the fact the in-plane dual-scale impregnation characteristics for a part at macro-level (for woven fabrics), can be estimated by employing a dual-scale flow modelling of a 9-ply UC. In dealing with a single-ply unit cell, the in-plane dual-scale permeability of multi-ply unit cells, comes to be higher with an increase ranging from $29.1 \%$ to $61.3 \%$, e.g., 20.9 and 30.5 $\left(\times 10^{-11} \mathrm{~m}^{2}\right)$ for nested 1-ply and 9-ply woven models, respectively. This emphasises the dual-scale flow simulations of a single-ply UC cannot be regarded as a RVE of multi-ply woven fabrics, but can only be used for a single woven layer of fibre preform.

Figure 10 represents pressure gradient contours for in-plane and through-thickness dual-scale permeabilities of the 15-ply woven model. In single-phase steady flow cases, the pressure gradient is important, since it can explain the flow behaviour and progression in-between and within the fibre bundles. As it can be seen from Fig. 10, higher pressure (or flow resistance) for the in-plane dual-scale permeability, is observed in nested and non-shifted multiple plies, while this becomes lower in oriented stacking plies. It stems from the fact the weft yarns $\left(90^{\circ}\right)$ resist the flow progression for nested and non-shifted plies, as being transverse to the flow direction. Similar through-thickness flow behaviour is observed for all different stacking sequences, owing to packing arrangement of fibres and/ or yarns, as being perpendicular to the flow advancement.

The results, furthermore, explain that flow patterns are critical, and can either promote or prevent voids. The induced voids are an unimpregnated areas (dry spots or unwetted fibres), and that are due to key factors, which profoundly rely on permeability. Fig. 8 shows lower permeabilities that a transverse flow (perpendicular to the fibres) will undergo high flow resistance and can potentially be responsible of void formation. This is unlike the axial flows (parallel to the fibres) that are provided with more permeable zones, in which the localised voids could merely occur at the flow front region. The presence of meso- or micro-voids in the final part impairs or may aggravate its mechanical properties in terms of flexural and compressive strength, and interlaminar shear strength [37, 38]. Thereby, an evolution of the dual-scale voidage in LCM during the filling process is significant, and this needs to be predicted to prevent such defects.

\subsection{In-Plane Flow Front Progression}

In order to test the validity of the determined RVE dual-scale permeabilities, a simple twodimensional (2D) macro-scale flow simulations of homogenous isotropic porous media in LCM is preformed. The developed multi-scale flow model allows the prediction of the filling time, which can be then compared against the analytical solution of one-dimensional (1D) Darcy's law (c.f., Appendix A) [33]. This was applied to 2D weave layers with boundary conditions and geometry details as illustrated by Fig. 11. A stabilising point is selected (e.g., 9-ply dual-scale permeability) for each woven fabric aggregate porosity, i.e., $40 \%, 50 \%$, and $60 \%$. The flow front simulations are performed on nested, non-shifted, and oriented cases, by employing the volume-of-fluid (VOF) method and adopting an implicit 

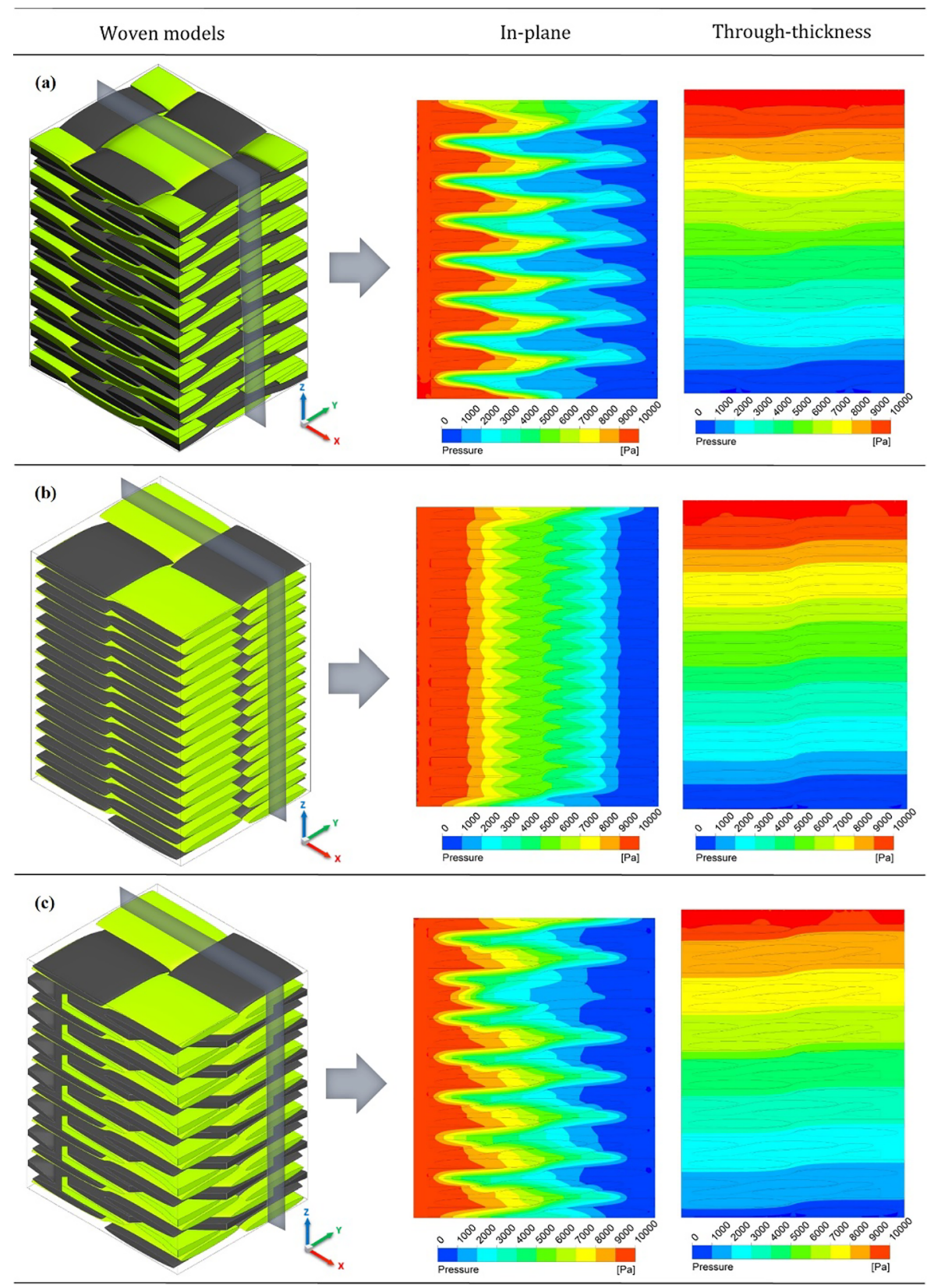

Fig. 10 Pressure gradient contours for in-plane and through-thickness dual-scale permeabilities of the 15-ply woven model: (a) nested, (b) non-shifted, and (c) oriented

time-stepping scheme. In this study, a number of tracking points are created and located in the direction of the flow advancement for reporting the flow front positions as a function of time. 


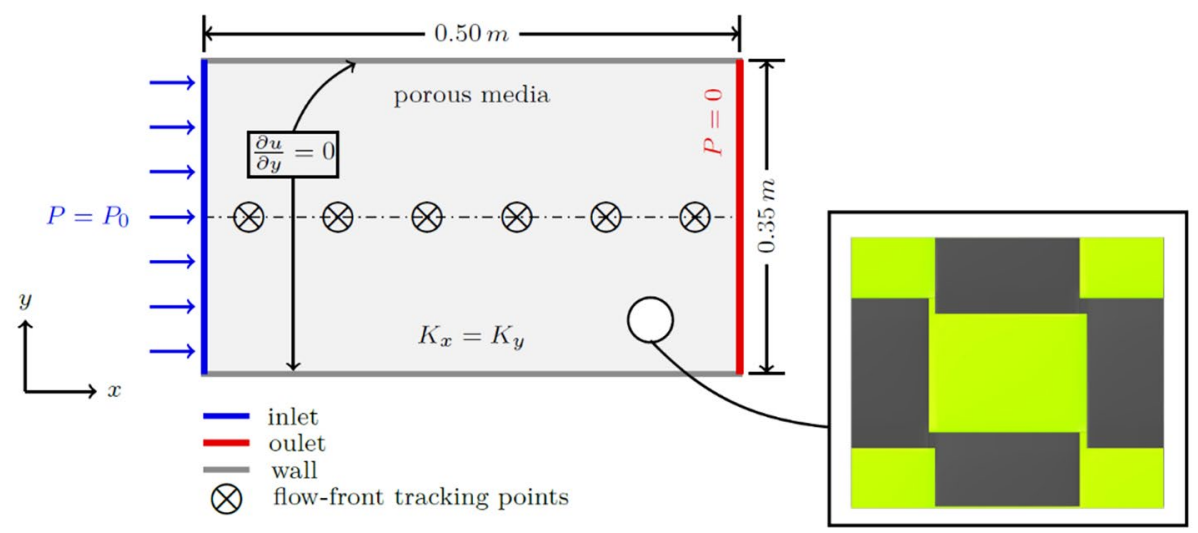

Fig. 11 Details of geometry and boundary conditions for multiple plies of 2D woven fabrics

Figure 12 shows a good fit of the numerical predictions to the ones calculated by the Darcy's analytical solution, highlighting the applicability of the proposed dual-scale (with multi plies) permeability at macro-scale flow. In Fig. 12, the fill time for the oriented case appears to be shorter than the nested and non-shifted ones, this stresses the fact that more flow passages ease the flow progression, and hence the complete wet-out of fibre preforms
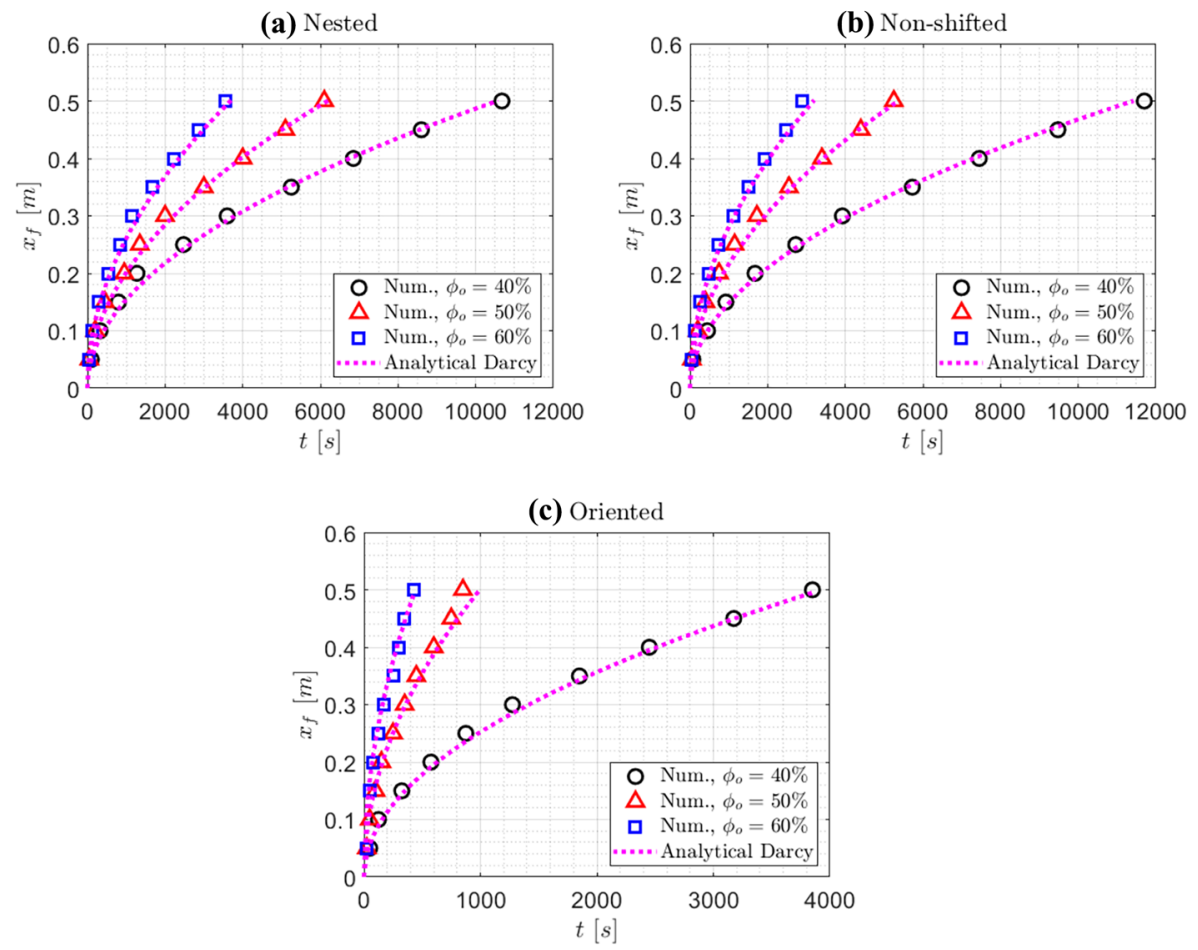

Fig. 12 Flow front predictions: (a) nested, (b) non-shifted, and (c) oriented 
occurs at shorter time. This measure, i.e., 2D rectilinear (channel) flow model, can then be extended to quantify three-dimensional viscous flow problems in simple or complex shaped composite parts during mould filling processes.

\section{Conclusions}

This paper investigated the role of upscaling in permeability modelling of woven fabric plies. A comparative analysis for single- and multi-ply woven fabric UCs with permeable tows $\left(\phi_{t}=20 \%\right)$ and various aggregate porosities $\left(\phi_{o}\right)$ of $40 \%, 50 \%$ and $60 \%$, was conducted to characterise transverse and longitudinal dual-scale flow. This involved the impact of different stacking methods such as nesting, non-shifting (zero-offset) and ply orientation.

The flow model used Stokes-Brinkman equations to simulate flows in the open (intertow) and porous (intra-tow) regions. Thus, the computed volume-averaged velocity was used to calculate the permeability tensor, i.e., a generalised form of Darcy's law. The determined micro-meso permeability was then inputted into a flow-front (macro-front) simulation of a 2D homogenous fibrous porous medium, in which a transient Darcy's solution can be employed to validate the multi-scale approach.

The results, first of all, depicted that because of more pathways offered by individual oriented off-axis plies $( \pm 45)$, the in-plane global permeability was one order of magnitude larger than the plate with $(0 / 90)$ woven layers.

The results, moreover, showed that dual-scale permeability modelling for the nested, non-shifted or oriented single-ply UC can be described by a RVE of a single-ply woven preform (meso-scale). However, with 5 UD plies the predicted dual-scale out-plane permeability can be used as RVE for macro-scale flow simulation. This becomes the same with 9 UD plies for in-plane permeability.

\section{Appendix A. Analytical Solution of Darcy's Equation}

In one dimensional Cartesian coordinates ( $x$-direction), Darcy's law combined with the continuity equation looks like this:

$$
\begin{gathered}
q=\frac{-\mathrm{K}}{\mu} \frac{d p}{d x} \\
\frac{d v}{d x}=0
\end{gathered}
$$

where $q$ is the superficial velocity of fluid, and it should be multiplied by the porosity, $\phi$, in order to get the actual velocity of the resin:

$$
q=v \cdot \phi=\frac{-\mathrm{K}}{\mu} \frac{d p}{d x}
$$

Assuming a constant pressure gradient, $d p / d x=\Delta p / x$, the velocity of a single point, e.g. the flow front can be written as $v=d x / d t$ : 


$$
\frac{d x}{d t}=\frac{-\mathrm{K}}{\phi \cdot \mu} \frac{\Delta p}{x}
$$

where $x$ is the position of the flow front relative to the inlet. Integrating the above equation will results in

$$
\begin{gathered}
\int_{0}^{x_{f}} x d x=\frac{-\mathrm{K}}{\phi \cdot \mu} \Delta p \int_{0}^{t} d t \\
\Rightarrow x_{f}^{2}=\frac{-2 \mathrm{~K}}{\phi \cdot \mu} \Delta p \cdot t
\end{gathered}
$$

This will result in the position of the flow front $x_{f}$ as a function of time $t$ :

$$
x_{f}(t)=\sqrt{\frac{-2 \mathrm{~K}}{\phi \cdot \mu} \Delta p \cdot t}
$$

Author Contribution Conceptualisation, H.A. and M.J.; Investigation, H.A.; Software, H.A.; Writing-original draft, H.A.; Writing-review and editing, H.A., M.J., C.A., and C.S.; Supervision; M.J.,C.A., and C.S. All authors have read and agreed to the published version of the manuscript.

Funding This research received no external funding.

Data Availability Data is contained within the article.

\section{Declarations}

Conflicts of Interest The authors declare no conflict of interest.

Open Access This article is licensed under a Creative Commons Attribution 4.0 International License, which permits use, sharing, adaptation, distribution and reproduction in any medium or format, as long as you give appropriate credit to the original author(s) and the source, provide a link to the Creative Commons licence, and indicate if changes were made. The images or other third party material in this article are included in the article's Creative Commons licence, unless indicated otherwise in a credit line to the material. If material is not included in the article's Creative Commons licence and your intended use is not permitted by statutory regulation or exceeds the permitted use, you will need to obtain permission directly from the copyright holder. To view a copy of this licence, visit http://creativecommons.org/licenses/by/4.0/.

\section{References}

1. Månson, J.A., Wakeman, M.D., Bernet, N.: Composite processing and manufacturing-an overview. (2000)

2. Advani, S., Sozer, E.: Liquid Molding of Thermoset Composites. Comprehensive Compos. Mater. 807-844 (2000)

3. Ermanni, P., Di Fratta, C., Trochu, F.: Molding: liquid composite molding (LCM). Wiley Ency. Compos. 1-10 (2011)

4. Tempelman, E., Shercliff, H., van Eyben, B.N.: Chapter 10 - Resin Transfer Molding. In: Tempelman, E., Shercliff, H., van Eyben, B.N. (eds.) Manufacturing and Design, pp. 171-186. Butterworth-Heinemann, Boston (2014) 
5. Pillai, K.M.: Governing equations for unsaturated flow through woven fiber mats. Part 1. Isothermal flows. Compos. Part A: Appl. Sci. Manuf. 33(7), 1007-1019 (2002)

6. Pillai, K.M.: Modeling the unsaturated flow in liquid composite molding processes: a review and some thoughts. J. Compos. Mater. 38(23), 2097-2118 (2004)

7. Gommer, F., Endruweit, A., Long, A.C.: Quantification of micro-scale variability in fibre bundles. Compos. A Appl. Sci. Manuf. 87, 131-137 (2016)

8. Tan, H., Pillai, K.M.: Multiscale modeling of unsaturated flow in dual-scale fiber preforms of liquid composite molding I: Isothermal flows. Compos. A Appl. Sci. Manuf. 43(1), 1-13 (2012)

9. Shojaei, A., Trochu, F., Ghaffarian, S.R., Karimian, S.M.H., Lessard, L.: An Experimental Study of Saturated and Unsaturated Permeabilities in Resin Transfer Molding Based on Unidirectional Flow Measurements. J. Reinf. Plast. Compos. 23(14), 1515-1536 (2004)

10. Schmachtenberg, E., Heide, J.S.Z., Töpker, J.: Application of ultrasonics for the process control of Resin Transfer Moulding (RTM). Polym. Testing 24(3), 330-338 (2005)

11. Carlone, P., Rubino, F., Paradiso, V., Tucci, F.: Multi-scale modeling and online monitoring of resin flow through dual-scale textiles in liquid composite molding processes. The International Journal of Advanced Manufacturing Technology 96(5-8), 2215-2230 (2018)

12. Drummond, J., Tahir, M.: Laminar viscous flow through regular arrays of parallel solid cylinders. Int. J. Multiph. Flow 10(5), 515-540 (1984)

13. Gebart, B.R.: Permeability of unidirectional reinforcements for RTM. J. Compos. Mater. 26(8), 11001133 (1992)

14. Cai, Z., Berdichevsky, A.L.: An improved self-consistent method for estimating the permeability of a fiber assembly. Polym. Compos. 14(4), 314-323 (1993)

15. Bruschke, M.V., Advani, S.G.: Flow of generalized Newtonian fluids across a periodic array of cylinders. J. Rheol. 37(3), 479-498 (1993)

16. Tamayol, A., Bahrami, M.: Transverse permeability of fibrous porous media. Phys. Rev. E. 83(4), 046314 (2011)

17. Song, Y., Chung, K., Kang, T., Youn, J.: Prediction of permeability tensor for three dimensional circular braided preform by applying a finite volume method to a unit cell. Compos. Sci. Technol. 64(10-11), 1629-1636 (2004)

18. Verleye, B., Croce, R., Griebel, M., Klitz, M., Lomov, S.V., Morren, G., Roose, D.: Permeability of textile reinforcements: Simulation, influence of shear and validation. Compos. Sci. Technol. 68(13), 2804-2810 (2008)

19. Tahir, M.W., Stig, F., Ảkermo, M., Hallström, S.: A numerical study of the influence from architecture on the permeability of 3D-woven fibre reinforcement. Compos. A Appl. Sci. Manuf. 74, 18-25 (2015)

20. Belov, E.B., Lomov, S.V., Verpoest, I., Peters, T., Roose, D., Parnas, R.S., Sol, H.: Modelling of permeability of textile reinforcements: lattice Boltzmann method. Compos. Sci. Technol. 64(7-8), 1069-1080 (2004)

21. Alotaibi, H., Jabbari, M., Soutis, C.: A Numerical Analysis of Resin Flow in Woven Fabrics: Effect of Local Tow Curvature on Dual-Scale Permeability. Materials 14(2), 405 (2021)

22. Papathanasiou, T.D.: Flow across structured fiber bundles: a dimensionless correlation. Int. J. Multiph. Flow 27(8), 1451-1461 (2001)

23. Wu, C.H., James Wang, T., James Lee, L.: Trans-plane fluid permeability measurement and its applications in liquid composite molding. Polym. Compos. 15(4), 289-298 (1994)

24. Okonkwo, K., Simacek, P., Advani, S.G., Parnas, R.S.: Characterization of 3D fiber preform permeability tensor in radial flow using an inverse algorithm based on sensors and simulation. Compos. A Appl. Sci. Manuf. 42(10), 1283-1292 (2011)

25. Fang, L., Jiang, J., Wang, J., Deng, C.: Effect of nesting on the out-of-plane permeability of unidirectional fabrics in resin transfer molding. Appl. Compos. Mater. 22(3), 231-249 (2015)

26. Scholz, S., Gillespie, J.W., Jr., Heider, D.: Measurement of transverse permeability using gaseous and liquid flow. Compos. A Appl. Sci. Manuf. 38(9), 2034-2040 (2007)

27. Mogavero, J., Advani, S.G.: Experimental investigation of flow through multi-layered preforms. Polym. Compos. 18(5), 649-655 (1997)

28. Calado, V.M., Advani, S.G.: Effective average permeability of multi-layer preforms in resin transfer molding. Compos. Sci. Technol. 56(5), 519-531 (1996)

29. Luce, T.L., Advani, S.G., Howard, J.G., Parnas, R.S.: Permeability characterization. Part 2: Flow behavior in multiple-layer preforms. Polym. Compos. 16(6), 446-458 (1995)

30. Jiang, J., Su, Y., Zhou, L., Guo, Q., Xu, C., Deng, G., Fang, L.: Effect of nesting on the permeability of multilayer unidirectional fabrics. Appl. Compos. Mater. 24(3), 625-642 (2017) 
31. Swery, E.E., Kelly, P.A., Allen, T.D., Bickerton, S.: Numerical Permeability Predictions of Woven Textiles: Examining the Characteristics of Multi-Layer Preforms. In ICCM 20: 20th Internat Conf Compos Mater. Copenhagen, Denmark (2015)

32. Sas, H.S., Wurtzel, E.B., Simacek, P., Advani, S.G.: Effect of relative ply orientation on the throughthickness permeability of unidirectional fabrics. Compos. Sci. Technol. 96, 116-121 (2014)

33. Yousaf, Z., Withers, P.J., Potluri, P.: Compaction, nesting and image based permeability analysis of multi-layer dry preforms by computed tomography (CT). Compos. Struct. 263, 113676 (2001)

34. Godbole, M.G., Gururaja, S., Joshi, M., Advani, S.: A semi-analytical model to characterize single ply and multiple plies UD-fabric permeability. Compos. Part A: Appl. Sci. Manuf. 136, 105951 (2020)

35. Yun, M., Carella, T., Simacek, P., Advani, S.: Stochastic modeling of through the thickness permeability variation in a fabric and its effect on void formation during Vacuum Assisted Resin Transfer Molding. Compos. Sci. Technol. 149, 100-107 (2017)

36. Bancora, S.P., Binetruy, C., Advani, S.G., Syerko, E., Comas-Cardona, S.: Effective permeability averaging scheme to address in-plane anisotropy effects in multi-layered preforms. Compos. A Appl. Sci. Manuf. 113, 359-369 (2018)

37. de Almeida, S.F.M., Neto, Z.D.S.N.: Effect of void content on the strength of composite laminates. Compos. Struct. 28(2), 139-148 (1994)

38. Bowles, K.J., Frimpong, S.: Void effects on the interlaminar shear strength of unidirectional graphitefiber-reinforced composites. J. Compos. Mater. 26(10), 1487-1509 (1992)

Publisher's Note Springer Nature remains neutral with regard to jurisdictional claims in published maps and institutional affiliations. 